\title{
An empirical research on the patterns and influencing factors of strategy formation processes
}

\author{
(C) Higher Education Press and Springer-Verlag 2008
}

\begin{abstract}
Using data collected from 126 firms, this article attempts to classify different patterns of strategy formation process and identify organizational factors influencing the processes. Based on a clustering analysis method, we identify four patterns of strategy formation processes varying in main characteristics, such as command, adaptive, vision, and external constraints, etc. We also find that a firm's ownership type and its development stage have significant effects on strategy formation processes.
\end{abstract}

Keywords strategy, strategy formation, strategy formation processes, strategy-making, clustering analysis

摘要 通过调查 126 家国内企业, 对企业战略形成过程进行分类, 并探索战略形成 过程差异的组织因素。采用聚类分析, 发现中国企业战略形成过程存在 4 种类型, 其主要特征表现在命令、适应、愿景、外部约束等方面, 同时发现：所有制类型、 企业发展阶段对企业的战略形成过程有显著影响。

关键词 战略, 战略形成, 战略形成模式, 战略制定, 聚类分析

\section{Introduction}

It is generally accepted that an effective strategy formation process (SFP) is of

Translated and revised from Nankai guanli pinglun 南开管理评论(Nankai Business Review), 2007, (10): 32-37

LI Yugang $(\bowtie)$, HU Junlian

School of Business, East China University of Science and Technology, Shanghai 200237, China

E-mail: yugangl@ecust.edu.cn 
decisive importance to timely strategy development. In the Chinese context, however, enterprise SFP has long been neglected by empirical studies. Up to now, it is not quite clear about how Chinese enterprises form their strategies, and what factors influence the process of strategy formation. This research aims to answer these questions. We plan to empirically identify different patterns and main characteristics of SFP and to present an initial analysis of the main influencing factors of SFP.

We first collected relevant strategy-making information from managers. After that, we tested our classification method of different patterns of SFP. Then using the clustering analysis approach, we classified sample companies' SFPs into four different patterns and summarized typical characteristics of each pattern accordingly. In the end, based on these patterns, we attempted to find out factors influencing enterprises' SFP.

\section{Theoretical background and hypotheses}

\subsection{Strategy formation process}

Numerous theoretical studies have been conducted on the types of enterprise strategy-making behaviors. Bourgeois and Brodwin (1984) proposed five different leadership styles in their study on strategy implementation. Their classification of leadership styles also revealed differences in strategy formation. Chaffee (1985) identified three strategy formation modes, namely linear mode, adaptive mode, and interpretive mode. Other strategy formation patterns include Mintzberg's (1978) three different enterprise SFPes (planning, adaptive and entrepreneurial types of strategy-making), Nonaka's (1988) middle-up-down mode (in contrast with traditional top-down and bottom-up modes), and Hart's (1992) command, symbolic, rational, transactive and generative modes. The notable work of Mintzberg (1999) proceeded to categorize the viewpoints jungle in the field of enterprise strategy formation into ten different schools, including the design school, the planning school, the positioning school, the entrepreneurial school, the cognitive school, the learning school, the power school, the cultural school, the environmental school, and the configuration school.

Other scholars empirically tested strategy-making modes (e.g. Fredrickson and Mitchell (1984), Miller and Friesen (1977, 1983), Shrivastava and Grant (1985), and Wooldridge and Floyd (1990). Based on extant literature, Bailey (2000) developed a scale for SFP measurement, consisting of six dimensions (namely planning, incrementalism, cultural, political, command and enforced choice) and 39 entries. Test using data from 5332 managers showed that the scale has acceptable credibility and reliability. Collier et al. (2000) adopted the said scale 
to analyze strategy making process with staff participation. In his article, Collier divided the SFP into items dimensions with 43 items, as shown in the right column of Fig.1.

$\underline{\text { Factors influencing SFP }}$

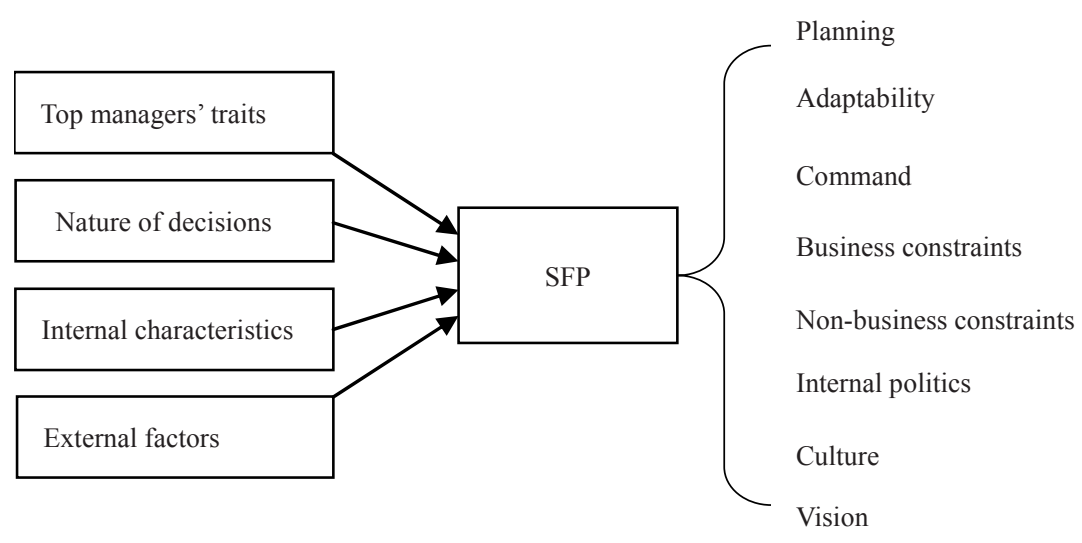

Fig. 1 Factors influencing SFP

Some domestic researchers have also studied on the process of Chinese enterprise strategy formation. For instance, using data collected from 75 valid questionnaires, Qi (2005) found that the top managers' dictatorial rules were common among Chinese enterprises, that is to say, the strategy-making are quite sporadic and characterized with brevity in many Chinese enterprises. Generally speaking, although there have been numerous domestic studies on Chinese enterprises' strategy-making, very few were carried out from the perspective of SFP.

Drawing on the above rationale, we propose the following hypothesis:

H1: The SFP of Chinese enterprises can be classified into different patterns.

\subsection{Factors influencing strategy formation process}

SFP is affected by an array of different factors, as shown in the left part in Fig.1.

Next is a brief analysis of the factors influencing SFP (detailed analysis of the characteristics of SFP is presented in the section of measurement method).

Personal traits of $C E O$ or other top managers. On studying the personal traits of CEO and top management teams, scholars have found that the traits of need for achievement, risk propensity, tenure, and education level exert impacts on SFP (Nahavandi and Malekzadeh, 1993). 
Nature of decisions. Numerous researches have been carried out on the effect of decision nature on SFP (see Dutton, et al, 1989; Cray, et al, 1991; Dean and Sharfman, 1993; Papadakis, Lioukas and Chambers, 1998). Fredrickson discovered that when a decision to be made is interpreted as a threat, rather than a chance, the SFP that followed is characterized by more rationality. Papadakis et al (1998) pointed out that most empirical studies only focused on a single decision trait (say, chance or threat) and its effect on SFP and a majority of existing researches have merely concentrated on the early stage of decision-making, that is, problem identification and diagnosis. They asserted that extant literature failed to unpack the black box of how does nature of decision itself help to shape SFP.

Internal characteristics of organization, including enterprise size, ownership type, internal control mechanism, and performance, etc. Child (1972) found that enterprise size affects the enterprise's decision-making framework. Consistent with this conclusion, Fredrickson and Iaquinto (1989) pointed out that the bigger an enterprise, the more rational its strategy-making process. However, Dean and Sharfman (1993) concluded that differences in strategic decision-making can not be attributed to enterprise size. Still other scholars proposed that the ownership structure and types of internal control mechanism have impact on strategic decision-making process (see Lioukas, et al, 1993). To illustrate, both Mintzberg (1973) and Mallory et al.(1983) reported that there are differences in the decision-making patterns between British domestic companies and transnational companies operating in Britain.

External environment factors. The environmental determinism presumes that SFP is an adaptation to both external opportunities and threats. The function of managerial personnel, therefore, is to facilitate this process of adaptation (see Lieberson and O'Connor, 1972; Hannan and Freeman, 1977). Studies taking the stance of this school, say Bourgeois (1988), Fredrickson and Iaquinto (1989), mostly focus on the dynamics and antagonism of environment (or industry). Yet no generally accepted conclusions have been achieved.

Considering the complexity of factors influencing organizational strategy-making, to ensure the operationablity of our study, we focus in this paper only on influencing factors of SFP at the organizational level, namely, the effects of an enterprise' ownership type, size, industry, and specific development stage on SFP.

Given these assumptions, we develop four hypotheses as follows:

H2: An enterprise's ownership type significantly affects its SFP.

H3: An enterprise's size significantly affects its SFP.

H4: The industry an enterprise is in significantly affects its SFP.

H5: The phase of life cycle an enterprise is in significantly affects its SFP. 


\section{Research methods and procedures}

\subsection{Data collection}

Study participants were MBA students (including students from senior manager classes and part-time MBA classes) from East China University of Science and Technology. A total of 135 questionnaires were delivered, of which 128 copies were returned. As a gauge, questionnaires with missing data were eliminated. A total of 126 valid questionnaires were deemed as valid and used as samples (with a valid return rate of $93 \%$ ). $79 \%$ of participants were senior or mid-level managers from various enterprises, implying that data collected from the survey can, to a large extent, reflect the real SFPs in their enterprises. Among the sampled enterprises, $16.7 \%$ were private-owned companies, $23.8 \%$ state-owned companies, $10.3 \%$ stock companies, $24.6 \%$ foreign-owned companies, $19.8 \%$ jointly-owned companies, and $4.8 \%$ other types of companies. As for enterprise size, $25.4 \%$ sample companies had more than 1000 employees, $40.5 \%$ between 100 to 1000 employees, $31 \%$ between 10 to 10 employees, and $3.2 \%$ had less than 10 employees. Industry distribution was: $45.2 \%$ sample companies were from manufacturing industry, $6.3 \%$ from trading, $9.5 \%$ from finance, $3.2 \%$ from energy and transportation, $11.1 \%$ from service, 9.5\% from IT, and $15.1 \%$ from other industries. As for enterprise development stages, $15.9 \%$ enterprises were at initial stage, 29.4 at growth stage, and 39.7 at maturity stage. This general description showed that the sampled companies in the present article were of satisfactory representativeness, thus could serve our study purpose well.

\subsection{Measurement}

(1) SFP. We adopted Collier's scale in measuring SFP. As noted, Collier extended Bailey's SFP scale into a new one with 47 items. Later, using principal component analysis and $\alpha$ factor analysis methods, Collier reduced the original 47-item scale to a 43-item one with 8 dimensions, namely planning, adaptive, command, internal politics, culture, vision, business constraints, and non-business constraints. Specifically, planning dimension emphasizes the importance of making strategic decisions rationally according to a set of pre-set procedures; adaptive dimension refers to the necessity of making timely adjustments to enterprise strategies gradually and flexibly as external environment requires; command dimension highlights the decisive importance of $\mathrm{CEO}$ or other important figure in strategy-making; internal politics dimension stresses the effects of conflicts and bargains among individuals or cliques in an organization on the organization's strategy choice; culture dimension accents the 
effects of an organization's past history, routine, and experience on the organization's strategy choice; vision dimension lays emphasis on the impacts an organization's future picture or shared value about the future on the organization's strategy direction; business constraints dimension emphasizes the influences from an enterprise's external business environment (exclude government or other government-related organizations); non-business constraints dimension refers to influences from outside organizations, such as government or other related organizations.

The reason why we adopted Collier's scale, instead of Bailey's scale in the present article was that Collier divided the compulsory choice in SFP into business constraints and non-business constraints, as well as set vision out as a new dimension, which we believe is more applicable in the Chinese context. We used the 43 items in Collier's scale to describe strategy-making process. Participants were required to judge the degree of conformity the SFC in their own enterprises with corresponding items in the questionnaire. The degree of conformity was measured with a 7-point Liker scale, ranging from 1 (does not match at all) to 7 (highly match).

To guarantee a high validity of the scale, we delivered 23 questionnaires as a trial test. According to the results of this trail test, we modified the expressions ways of some questions in the original scale. Three experts were invited to evaluate the modified scale and entries with problems were modified once more. Credibility of the modified scale was shown in Table 1.

Table 1 Reliability test of the modified Collier scale

\begin{tabular}{lccc}
\hline Variables & Number of questions & $\alpha$ & Standard $\alpha$ \\
\hline Planning & 7 & 0.9010 & 0.9020 \\
Adaptive & 6 & 0.8182 & 0.8189 \\
Command & 5 & 0.8411 & 0.8384 \\
Business constraints & 4 & 0.7401 & 0.7399 \\
Non-business constraints & 3 & 0.7546 & 0.7618 \\
Internal politics & 6 & 0.6758 & 0.6750 \\
Culture & 7 & 0.7991 & 0.8054 \\
Vision & 5 & 0.8679 & 0.8697 \\
\hline
\end{tabular}

(2) Types of enterprise ownership, including private-owned, state owned, stock, foreign-owned, joint-stock and other types of companies.

(3) Enterprise scale. The variable of enterprise scale was presented by the number of employees in an enterprise. As the participants of our study were on a MBA program and unable to tell the exact number of employees in their enterprise currently, we divided enterprise scale into four ranges, namely "less than 10 employees", "10-1000 employees", "100-1000 employees" and "more than 1000 employees". 
(4) Industry. Sampled companies in the current study were from 7 industries, including manufacturing, trading, finance, energy and transportation, service, IT, and other industries.

(5) Enterprise life cycle stage. We divided the life cycle stages of the sampled companies as follows: initial stage, fast growth stage, maturity stage, and transformation stage.

\section{Results}

\subsection{Scale validity}

As mentioned earlier, Collier used the methods of principal components analysis and alpha factoring when developing his scale. Due to sample limitations, we did not attempt to develop a brand new scale. Thus we did not conduct a principal component analysis. What we interested was whether Collier's scale (with 8 dimensions and 43 items) has a high internal reliability so that it can be used for our study purpose.

SPSS software package was used to test the reliability of Collier's Scale. Results (as shown in Table 1) that except the Cronbach $\alpha$ of internal politics dimension was $0.68, \alpha$ coefficients of other dimensions were all considerably higher than 0.7 .

Devellis (1991) pointed out that $\alpha$ value between 0.65 and 0.7 is acceptable. We thus regarded the modified Collier's scale as having good internal reliability and can be used in the current study. In the sections that followed, the mean of several items under every dimension was used to represent an enterprise's final points on that dimension.

\subsection{Clustering analysis of strategy formation process}

Standard deviations of all samples in the eight dimensions are depicted in Table 2.

Table 2 Standard deviations of sample companies on the eight dimensions

\begin{tabular}{|c|c|c|c|c|c|c|c|c|}
\hline Variables & Planning & Adaptive & Command & $\begin{array}{l}\text { Business } \\
\text { constraints }\end{array}$ & $\begin{array}{c}\text { Non-business } \\
\text { constraints }\end{array}$ & $\begin{array}{l}\text { Internal } \\
\text { politics }\end{array}$ & Culture & Vision \\
\hline Variances & 1.70 & 1.27 & 2.45 & 1.32 & 2.20 & 1.08 & 1.02 & 1.60 \\
\hline
\end{tabular}

As a rule, the higher the standard deviation, the bigger the variances sample data from the means. Table 2 shows that sample had greatest variance in the dimension of command, and smallest variance in the dimension of culture.

By using the K-means clustering algorithm, we calculated separately sums of 
variances between different clusters, as depicted in Table 3 and in Fig. 2.

Table 3 Sums of intra-cluster variances under different clustering methods

\begin{tabular}{ccccccccc}
\hline $\begin{array}{c}\text { Number of } \\
\text { clusters }\end{array}$ & 1 & 2 & 3 & 4 & 5 & 6 & 7 \\
\hline $\begin{array}{c}\text { Sums of } \\
\text { intra-cluster } \\
\text { variances }\end{array}$ & 1580.71 & 1303.83 & 1100.46 & 988.51 & 955.19 & 892.39 & 829.06 \\
\hline
\end{tabular}

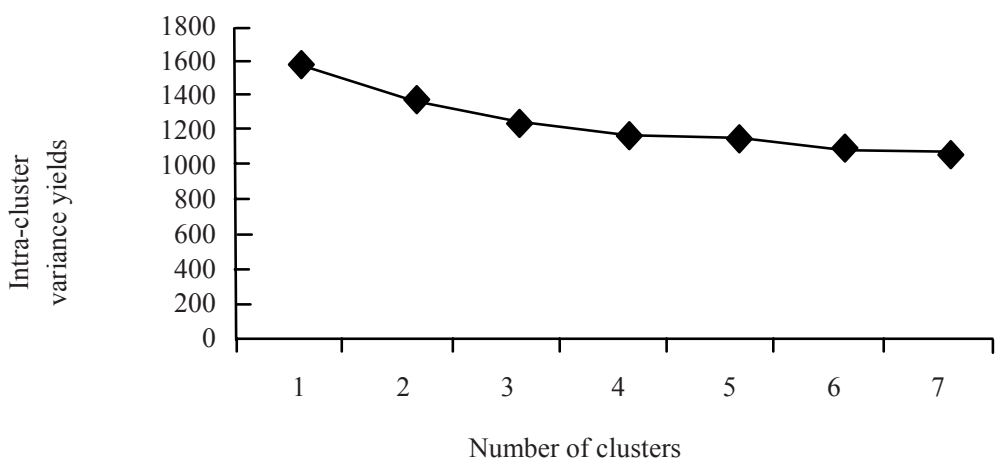

Fig. 2 Sum of intra-cluster variance under different clustering methods

Fig. 2 depicts that when the number of clusters is increased from 2 to 4 , sum of intra-cluster variances decreases pretty quickly. With increases in cluster numbers, the decrease of sum of intra-cluster variances slows down. Hence we divided the total samples into 4 clusters in accordance with the SFP characteristics of each cluster.

After clustering, the number of sample companies in each pattern is shown in Table 4.

Table 4 Distribution of number of samples in the four SFP patterns

\begin{tabular}{lcc}
\hline Clusters & Number of samples & Percentage to total samples (\%) \\
\hline Pattern 1 & 19 & 15.08 \\
Pattern 2 & 32 & 25.40 \\
Pattern 3 & 38 & 30.16 \\
Pattern 4 & 37 & 29.37 \\
\hline
\end{tabular}

When divided into four patterns, we conducted significance tests on different characteristics of each SFP, as demonstrated in Table 5. 
Table 5 Single factor analysis of variance of four patterns on different dimensions

\begin{tabular}{lcccccc}
\hline & Cluster & \multicolumn{7}{c}{ Error } & $F$ & Sig. \\
\hline & Mean square & $\mathrm{d} f$ & Mean square & $\mathrm{d} f$ & & \\
Planning & 29.321 & 3 & 1.025 & 122 & 28.597 & 0.000 \\
Adaptive & 13.331 & 3 & 0.976 & 122 & 13.664 & 0.000 \\
Command & 57.310 & 3 & 1.097 & 122 & 52.228 & 0.000 \\
Business constraints & 11.813 & 3 & 1.066 & 122 & 11.077 & 0.000 \\
Non-business constraints & 44.675 & 3 & 1.158 & 122 & 38.591 & 0.000 \\
Internal politics & 9.371 & 3 & 0.876 & 122 & 10.695 & 0.000 \\
Culture & 3.033 & 3 & 0.968 & 122 & 3.134 & 0.028 \\
Vision & 28.551 & 3 & 0.936 & 122 & 30.502 & 0.000 \\
\hline
\end{tabular}

As shown, the significance possibilities of all patterns in each dimension were all smaller than 0.5 , indicating that the means of each pattern were significant different in each of the eight dimensions. Thus H1 was supported, that is, enterprises vary significant in their SFPs.

To compare the differences of each pattern in SFP and to depict their characteristics, we calculated the means of all four patterns on each dimension, as shown in Table 6.

Table 6 Means of the four clusters in each dimension

\begin{tabular}{ccccccccc}
\hline Clusters & Planning & Adaptive & Command & $\begin{array}{c}\text { Business } \\
\text { constraints }\end{array}$ & $\begin{array}{c}\text { Non-business } \\
\text { constraints }\end{array}$ & $\begin{array}{c}\text { Internal } \\
\text { politics }\end{array}$ & Culture & Vision \\
\hline Pattern 1 & 2.07 & 3.26 & 5.22 & 5.03 & 4.11 & 3.98 & 4.34 & 2.74 \\
Pattern 2 & 4.23 & 4.51 & 5.40 & 4.77 & 2.80 & 3.83 & 4.89 & 5.21 \\
Pattern 3 & 4.34 & 5.00 & 3.92 & 5.28 & 5.29 & 3.70 & 4.71 & 4.83 \\
Pattern 4 & 4.55 & 4.19 & 2.52 & 3.96 & 3.14 & 2.79 & 4.24 & 5.02 \\
\hline
\end{tabular}

Table 6 indicates that the main characteristics of the Pattern 1 are command and business constraints, that is to say, strategies are made by top managers and there is a "top-down" mode of SFP. Meanwhile, as enterprise strategies are strictly constrained by external business environment, entrepreneurs in this pattern have to make their choices among several available strategies. Pattern 2 is characterized by dimensions of command and vision. Strategies in this pattern were made by top managers and were consistent with these enterprises' specific visions. The most prominent characteristics of Pattern 3 are adaptability and external constraints (including both business and non-business constraints), enterprises in this cluster constantly and gradually adjust their strategies in accordance with market changes and their strategies are constrained by external business and non business (such as government or other government-related organizations environment. In Pattern 4, an enterprise's strategy is an embodiment of the vision of its own. 
What needs to be notified is that the command dimension was negatively related to the vision dimension in the total sample. Yet in Pattern 3, we found the coefficient of correlation between the two was positive (not significant). Though from a statistical point of view, this might happen by accident. Still another explanation is that, an enterprise's vision made by a visionary type of leader is widely accepted by its employees, thus there could be a positive relation between the dimensions of command and vision.

\subsection{Analysis on the factors causing differences in strategy formation process}

As above, we identified four patterns of SFP. Next, we want to find out factors attributing to these different SFPs.

\subsubsection{Effects of types of enterprise ownership on SFP}

For convenience of sample distribution description and Chi-square testing, we divided the 120 sample companies (6 companies were eliminated from the sample for failing to meet the classification requirements) into two sub-groups, namely companies having foreign investors (including foreign-owned and jointly-invested companies) and domestic companies (including private-owned, state-owned, and stock companies). The distribution of these two sub-groups is depicted in Table 7.

Table 7 Distribution of the number of enterprises with different types of ownership in each SFP pattern

\begin{tabular}{lcc}
\hline & Domestic companies & $\begin{array}{c}\text { Companies having foreign } \\
\text { investors }\end{array}$ \\
\hline Pattern 1 & 13 & 6 \\
Pattern 2 & 14 & 18 \\
Pattern 3 & 23 & 10 \\
Pattern 4 & 14 & 22 \\
\hline
\end{tabular}

Table 8 shows the results of Chi-square test for ownership types.

Table 8 Chi-square test for types of ownership

\begin{tabular}{lccc}
\hline & Value & $\mathrm{d} f$ & Asymp. Sig. (2-sided) \\
\hline Pearson chi-square & 9.487 & 3 & 0.023 \\
Likelihood ratio & 9.664 & 3 & 0.022 \\
Linear-by-linear association & 1.835 & 1 & 0.176 \\
$N$ of valid cases & 120 & & \\
\hline
\end{tabular}

$N$ of valid cases

Notes: (1) 0 unit has an expected frequency smaller than 5.

(2) The minimum expected frequency is 8.87. 
Since the $p$ value of 2-sided asymp significance was smaller than 0.05 , H2 was supported, that is, there was significant difference in the SFPs between companies with foreign investors and domestic companies. Table 8 also shows that most Chinese companies cluster in Pattern 3, implying their SFPs are heavily constrained by external factors (adaptive dimension), while companies with foreign investors tend to cluster in Pattern 4 (vision dimension).

\subsubsection{Effects of enterprise size on SFP}

For convenience of sample distribution description and Chi-square testing, we analyzed only three types of enterprise sizes, as shown in Table 9.

Table 9 Distribution of number of different-sized enterprises in each SFP pattern

\begin{tabular}{lccc}
\hline & $10-100$ employees & $100-1000$ employees & More than 1000 employees \\
\hline Pattern 1 & 7 & 6 & 6 \\
Pattern 2 & 16 & 11 & 5 \\
Pattern 3 & 14 & 16 & 8 \\
Pattern 4 & 6 & 18 & 13 \\
\hline
\end{tabular}

Table 10 shows the results of Chi-square test for enterprise size.

Table 10 Chi-square test for enterprise size

\begin{tabular}{lccc}
\hline & Value & $\mathrm{d} f$ & Asymp. Sig. (2-sided) \\
\hline Pearson chi-square & 10.418 & 6 & 0.108 \\
Likelihood ratio & 11.013 & 6 & 0.088 \\
Linear-by-linear association & 3.628 & 1 & 0.057 \\
$N$ of valid cases & 126 & & \\
\hline
\end{tabular}

Notes: (1) One unit (8.3\%) has an expected frequency smaller than 5.

(2) The minimum expected frequency is 4.83 .

Since the $p$ value of 2-sided asymp significance was bigger than 0.05 , H3 was not supported, that is, there were no significant differences in the SFPs of different-sized enterprises.

\subsubsection{Industrial influences on SFP}

We were unable to test $\mathrm{H} 4$ due to the following two reasons: first, the sample distribution could not meet the requirement for Chi-square test; second, we failed to find a satisfactory theoretical basis to combine different industries into one. 


\subsubsection{Effects of an enterprise's specific development stage on SFP}

For convenience of sample distribution description and Chi-square testing, we merged the initial stage and fast growth stage as one. Distribution of the number of enterprises at these three different development stages are shown as below.

Table 11 Distribution of the number of enterprises at different stage of development in each SFP pattern

\begin{tabular}{lccc}
\hline & Initial and fast growth stage & Maturity stage & Transformation stage \\
\hline Pattern 1 & 4 & 9 & 6 \\
Pattern 2 & 22 & 8 & 2 \\
Pattern 3 & 15 & 18 & 5 \\
Pattern 4 & 16 & 15 & 6 \\
\hline
\end{tabular}

Table 12 shows the results of Chi-square test for different development stages in an enterprise's life cycle.

Table 12 Chi-square test for different enterprise development stages

\begin{tabular}{lccc}
\hline & Value & $\mathrm{d} f$ & Asymp. Sig. (2-sided) \\
\hline Pearson chi-square & 14.482 & 6 & 0.025 \\
Likelihood ratio & 14.430 & 6 & 0.025 \\
Linear-by-linear association & 0.222 & 1 & 0.638 \\
$N$ of valid cases & 126 & & \\
\hline
\end{tabular}

Notes: (1) Two units (16.7\%) have expected frequencies smaller than 5.

(2) The minimum expected frequency is 2.87.

Since the $p$ value of 2-sided asymp significance was smaller than $0.05, \mathrm{H} 3$ was supported, that is, there were significant differences in the SFPs in enterprises at different development stages. Specifically, most enterprises at the initial and fast growth stages belong to Pattern 2 and are characterized by command and vision dimensions, while enterprises at the maturity stage are characterized by business and non-business constraints dimensions (Pattern 3).

\section{Discussion}

\subsection{Conclusion and practical implications}

The current study attempted to identify major patterns of SFP in Chinese enterprises. As the scale we adopted was originally developed by western scholars in the British context, we first of all tested the inner conformity of the scale to decide whether it could be use in China. 
We found no enterprises, regardless of the ownership types, had points bigger than five in the dimensions of planning, culture, and inner politics. Possible explanations are: first, low points in the dimension of planning indicate that Chinese enterprises are not efficient at strategy-making, which is consistent with our empirical observations. As a matter of fact, most Chinese enterprises do not have effective and standardized strategy-making procedures. As for companies with foreign investors involved, since most of these companies' strategic decision-making centers are located outside China, their Chinese branch companies only have very little strategy making authorities, hence scored low on the dimension of planning; second, low scores in the dimensions of culture and inner politics. As China is currently in an economic transition period, employee turnover rate tend to be very high. Thus though there are certain shared cultures in Chinese enterprises, these cultures may not be overwhelmingly dominant, resulting in a weak effect the dimension of enterprise culture on SFP.

We also found a huge difference in SFPs among Chinese enterprises. Based on our analysis, we identified four patterns of SFPs, namely, Command and Business Constraints, Command \& Vision, Adaptive \& External Constraints, and Vision. As a whole, the most predominant characteristics of these four patterns are command, adaptive, vision and constraints.

Practical implications include: first, we proved that strategy measuring scale developed by western scholars can also used to describe and interpret the SFP of Chinese enterprises; second, there are huge differences in the SFPs of different enterprises, thus when improving strategy-making efficiency, different approaches should be adopted; third, we found that Chinese enterprises are quite weak at systematically planning their strategies. Though the routinization of strategy-making process in the western companies were seriously criticized by Mintzberg (1987), we argue that most Chinese enterprise still need more rationality and standardized procedures when making their strategies.

\subsection{Limitations and future research directions}

This research has two limitations. First, in the process of SFP measuring, data was collected from the subjective judges of a single manager, which may not reflect real situations in his/her companies sometimes. Second, due to sample limitations, we measured the factors influencing SFP only at an organizational level. Therefore, further study with more samples needs to be given to test the stability of our conclusions.

Future research directions include: first, there are big differences in the SFPs in Chinese enterprises, more researches are needed to further explore the main characteristics in SFPs in different types of enterprises; second, as there are an array of factors influencing SFP, it is necessary to identify the key influencing 
factor and its function mechanism by means of case study or empirical test.

\section{References}

Bailey A(2000). Validation of a multi-dimensional measure of strategy development processes. British Journal of Management, 11(2): 151-162

Bourgeois L J, Brodwin D(1984). Strategic implementation: Five approaches to an elusive phenomenon. Strategic Management Journal, (5): 241-264

Bourgeois L J, Eisenhardt K M(1988). Strategic decision processes in high velocity environments: Four cases in the microcomputer industry. Management Science, 34(7): $816-835$

Chaffee E(1985). Three modes of strategy. Academy of Management Review, (10): 89-98

Child J(1972). Organizational structure, environment and performance: The role of strategic choice. Sociology, (6): 1-22

Collier N, Fishwick F, Floyd S W(2004). Managerial involvement and perceptions of strategy process. Long Range Planning, 37(1): 67-83

Cray D, Mallory G R, Butler R J, Hickson D J, Wilson D C(1991). Explaining decision processes. Journal of Management Studies, 28(3): 227-251

De Vellis R F(1991). Scale Development: Theory and Applications. Newbury Park, CA: Sage Publications

Dean J W, Sharfman M P(1993). Procedural rationality in the strategic decision making process. Journal of Management Studies, 30(3): 587-610

Dutton J E, Walton E J, Abrahamson E(1989). Important dimensions of strategic issues: Separating the wheat from the chaff. Journal of Management Studies, 26(4): 279-296

Fredrickson J W(1985). Effects of decision motive and organizational performance level on strategic decision processes. Academy of Management Journal, 28(4): 821-843

Fredrickson J W, Iaquinto A L(1989). Inertia and creeping rationality in strategic decision processes. Academy of Management Journal, 32(4): 516-542

Fredrickson W, Mitchell R(1984). Strategic decision processes: comprehensiveness and performance in an industry with an unstable environment. Academy of Management Journal, 27(2): 399-323

Hannan M T, Freeman J H(1977). The population ecology of organizations. American Journal of Sociology, (82): 929-964

Hart S L(1992). An integrative framework for strategy-making processes. Academy of Management Review, (17): 327-351

Lewin A Y, Stephens C U(1994). CEO attitudes as determinants of organization design: an integrated model. Organization Studies, 15(2): 183-212

Lieberson S, O'Connor J F(1972). Leadership and organizational performance: a study of large corporations. American Sociological Review, (37): 117-130

Lioukas S, Bourantas D, Papadakis V(1993). Managerial autonomy of state-owned enterprises: Determining factors. Organization Science, 4(4): 645-666

Mallory G R, Butler R J, Cray D, Hickson D J, Wilson D C(1983). Implanted decision-making: American owned firms in Britain. Journal of Management Studies, 20(2): 191-211

Miller D, Friesen P H(1977). Strategy-making in context: the empirical archetypes. Journal of Management Studies, 14(3): 253-280

Miller D, Friesen P H(1983). Strategy-making and environment: The third link. Strategic 
Management Journal, 4(3): 221-235

Miller D, Toulouse J M(1986). Chief executive personality and corporate structure in small firms. Management Science, (32): 1389-1409

Mintzberg H(1973). Strategy making in three modes. California Management Review, 16(2): $44-53$

Mintzberg H(1978). Patterns in strategy formation. Management Science, (24): 934-948

Mintzberg H(1987). The fall and rise of strategic planning. Harvard Business Review, 65(1): $107-114$

Mintzberg H, Lampel J(1999). Reflecting on the strategy process. Sloan Management Review, 40(3): 21-30

Nahavandi A, Malekzadeh A R(1993). Leader style in strategy and organizational performance: an integrative framework. Journal of Management Studies, 30(3): 405-425

Nonaka I(1988). Toward middle-up-down management: accelerating information creation. Sloan Management Review, (29): 9-18

Papadakis V, Lioukas S, Chambers D(1998). Strategic decision making processes: the role of management and context. Strategic Management Journal, (9): 115-132

Qi Daqing(2005). Strategy setting and enforcing at Chinese businesses. China New Time, (1): 50-51(in Chinese)

Shrivastava P, Grant J H(1985). Empirically derived models of strategic decision-making processes. Strategic Management Journal, 6(2): 97-113

Wooldridge B, Floyd S W(1990). Strategy process, middle management involvement, and organizational performance. Strategic Management Journal, 11(3): 231-241 